\title{
From intracardiac electrograms to electrocardiograms. Models and metamodels
}

\author{
G. Ebrard ${ }^{1}$, M. A. Fernández ${ }^{1}$, J.-F. Gerbeau ${ }^{1}$, F. Rossi $^{2}$, N. Zemzemi ${ }^{1,3}$ \\ 1 INRIA Paris-Rocquencourt BP 105, F-78153 Le Chesnay Cedex, France \\ 2 Institut TELECOM, TELECOM ParisTech, LTCI - UMR CNRS 5141, 46, rue Barrault, F-75013 Paris. \\ 3 Université Paris 11, Laboratoire de mathématiques d'Orsay, Bâtiment 425, F-91405 Orsay Cedex, France
}

\begin{abstract}
We consider the problem of building a standard electrocardiogram (ECG) from the electrical potential provided by a pacemaker in a few points of the heart (electrogram). We use a 3D mathematical model of the heart and the torso electrical activity, able to generate "computational ECG", and a "metamodel" based on a kernel ridge regression. The input of the metamodel is the electrogram, its output is the ECG. The 3D model is used to train and test the metamodel. We illustrate the performance of the proposed strategy on simulated bundle branch blocks of various severities and a few clinical data.
\end{abstract}

\section{Introduction}

In this work, we show how an ECG simulation tool can be used to address an industrial problem raised by a pacemaker manufacturer. A typical modern pacemaker with implantable defibrillator can provide the measurements of the electrical potential in several points of the heart. Instead of these raw data, the pacemaker manufacturer would like to provide the clinician with something more familiar, namely, a standard electrocardiogram (ECG). Of course, given the small number of measurements in the heart, this "pseudo-ECG" is not expected to be very accurate. The idea is rather to get a rough instantaneous ECG, just by remotely questioning the pacemaker, without setting up all the leads needed to get a real standard ECG. Of course, this cannot replace a genuine ECG but since the data are available for free, one can find it interesting to use them for a simple routine clinical examination.

To formalize a little bit the problem at hand, we denote by $x(t) \in \mathbb{R}^{m_{P}}$ the vector representing the intracardiac potential measured by the pacemaker in $m_{p}$ points in the heart at time $t$. A typical value for $m_{P}$ is five. The quantity $x(t)$ will be called an electrogram (EGM). We denote by $y(t)$ a lead of a standard ECG, for example the first lead, known as DI, which is the difference of potential between the left and the right arms. The data $(x(t), y(t))_{t \in T}$ define a training set. With the training set, we try to build a function $f$ such that $y(t) \approx f(x(t)$ ) (as a matter of fact the argument of $f$ will be slightly more complicated, see below). After a while, say a few months later, the same patient will probably have a different EGM $\tilde{x}(t)$. Nonetheless, $\tilde{y}(t)=f(\tilde{x}(t))$ is expected to give a good approximation of the first lead of the ECG. The purpose of this work is to propose a possible approach to actually build function $f$.

The construction of a function $f$ that satisfies $y(t) \approx f(x(t))$ on the training set is a relatively easy task. The difficulty is to build an $f$ able to reconstruct a good ECG $\tilde{y}$ from an EGM $\tilde{x}$ which is not in the training set. Several methods have been developed in the machine learning community to address this kind of problem. In this work, we propose to use the so-called kernel ridge regression and a simple treatment of the time series defined by the EGM. We present this method in $\S 3$.

Another difficulty of the problem is that the function $f$ has to be patient specific (for the obvious reason for example that the location of the points where the EGM is measured varies from a patient to another). The training set corresponds to a certain state of the patient and it is not easy to test the reconstruction algorithm on different situations. Another contribution of this work is to show that 
a numerical simulation tool can be convenient to address this issue. In $\S 2$, we briefly present this simulation tool. Of course, many simplifications are done to model the complex electrophysiology of the heart in a tractable way. Nevertheless, in spite of these simplifications, the model has been built in order to provide realistic ECGs. It is therefore a good candidate to test the reconstruction algorithm, as shown in $\S 4$.

\section{$2 \quad$ Model}

The mathematical model used to generate the computational ECGs has been presented in other works $([1,2])$ but to keep this paper as self contained as possible, we briefly recall it in this section.

We denote by $\Omega_{\mathrm{T}}$ a $3 \mathrm{D}$ domain representing the torso and by $\Omega_{\mathrm{H}}$ a $3 \mathrm{D}$ domain representing the heart. The model consists of two degenerate parabolic reaction-diffusion partial differential equations (PDE), known as the bidomain equations (see e.g. $[7,11]$ ), coupled to a system of ordinary differential equations (ODE):

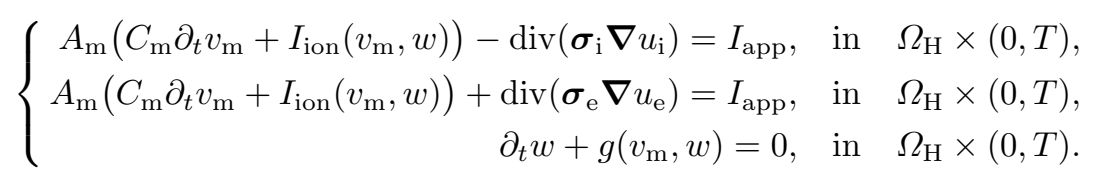

The two PDEs describe the dynamics of the averaged intra- and extracellular potentials $u_{\mathrm{i}}$ and $u_{\mathrm{e}}$, whereas the ODE is related to the dynamics of the myocardium cell membrane. The unknown $w$ represents the ion concentrations and gating variables, $v_{\mathrm{m}}=u_{\mathrm{i}}-u_{\mathrm{e}}$ is the transmembrane potential, $C_{\mathrm{m}}$ is the membrane capacitance, $A_{\mathrm{m}}$ stands for the volume density of membrane surface, $\boldsymbol{\sigma}_{\mathrm{i}}, \boldsymbol{\sigma}_{\mathrm{e}}$ are the intra- and extracellular conductivity tensors and $I_{\text {app }}$ is an external applied volume current. The potentials are defined up to an additive constant. We get rid of this undetermination by enforcing a zero mean value to $u_{\mathrm{e}}$.

The nonlinear reaction term $I_{\mathrm{ion}}\left(v_{\mathrm{m}}, w\right)$ and the function $g\left(v_{\mathrm{m}}, w\right)$ depend on the ionic model. Several ionic models have been proposed for decades, either based on physiological or phenomenological considerations (see $[7,11]$ for a review). In our works, we use the Mitchell-Schaeffer equations [4] which is a two-current phenomenological model. The unknown $w$, which is scalar is this case, is a gate variable. This model is defined by

$$
\begin{aligned}
& I_{\mathrm{ion}}\left(v_{\mathrm{m}}, w\right)=-\frac{w}{\tau_{\text {in }}} v_{\mathrm{m}}^{2}\left(1-v_{\mathrm{m}}\right)+\frac{v_{\mathrm{m}}}{\tau_{\text {out }}}, \\
& g\left(v_{\mathrm{m}}, w\right)= \begin{cases}\frac{w-1}{\tau_{\text {open }}} & \text { if } v_{\mathrm{m}}<V_{\text {gate }}, \\
\frac{w}{\tau_{\text {close }}} & \text { if } v_{\mathrm{m}}>V_{\text {gate }},\end{cases}
\end{aligned}
$$

where $\tau_{\text {in }}, \tau_{\text {out }}, \tau_{\text {open }}, \tau_{\text {close }}$ and $V_{\text {gate }}$ are given parameters. The complexity of the Mitchell-Schaeffer ionic model is comparable to the well-known FitzHugh-Nagumo equations [5] but it is more convenient to parametrize and gives more realistic results for cardiac cells. It has been shown to be well-suited to obtain computational ECG [1].

The numerical simulation of the ECG signals, which are measurements of voltages (i.e. potential differences) on the body surface [3], requires a description of how the surface potential is related to the electrical activity of the heart. Such as description is based on the coupling of (1) with a diffusion equation in $\Omega_{\mathrm{T}}$ :

$$
\operatorname{div}\left(\boldsymbol{\sigma}_{\mathrm{T}} \boldsymbol{\nabla} u_{\mathrm{T}}\right)=0, \quad \text { in } \quad \Omega_{\mathrm{T}},
$$

where $u_{\mathrm{T}}$ stands for the torso potential and $\boldsymbol{\sigma}_{\mathrm{T}}$ for the conductivity tensor of the torso tissue. The boundary $\Gamma_{\text {ext }}$ can be supposed to be insulated, which corresponds to the condition

$$
\boldsymbol{\sigma}_{\mathrm{T}} \boldsymbol{\nabla} u_{\mathrm{T}} \cdot \boldsymbol{n}_{\mathrm{T}}=0 \text { on } \Gamma_{\mathrm{ext}},
$$


where $\boldsymbol{n}_{\mathrm{T}}$ stands for the outward unit normal on $\Gamma_{\text {ext }}$.

The following coupling conditions between (1) and (4) are enforced on the interface $\Sigma$ separating the heart $\Omega_{\mathrm{H}}$ and the torso $\Omega_{\mathrm{T}}$ :

$$
\left\{\begin{aligned}
u_{\mathrm{e}} & =u_{\mathrm{T}}, & & \text { on } \quad \Sigma, \\
\boldsymbol{\sigma}_{\mathrm{e}} \boldsymbol{\nabla} u_{\mathrm{e}} \cdot \boldsymbol{n} & =\boldsymbol{\sigma}_{\mathrm{T}} \boldsymbol{\nabla} u_{T} \cdot \boldsymbol{n}, & & \text { on } \quad \Sigma .
\end{aligned}\right.
$$

These relations can be simplified replacing the second equation by $\boldsymbol{\sigma}_{\mathrm{e}} \boldsymbol{\nabla} u_{\mathrm{e}} \cdot \boldsymbol{n}=0$ on $\Sigma$. This does not affect dramatically the ECG and saves a lot of computational time. In this work, all the tests are performed with this simplification. In Fig. 1 is given an example of ECG obtained with this model. For futher details, we refer to [1].
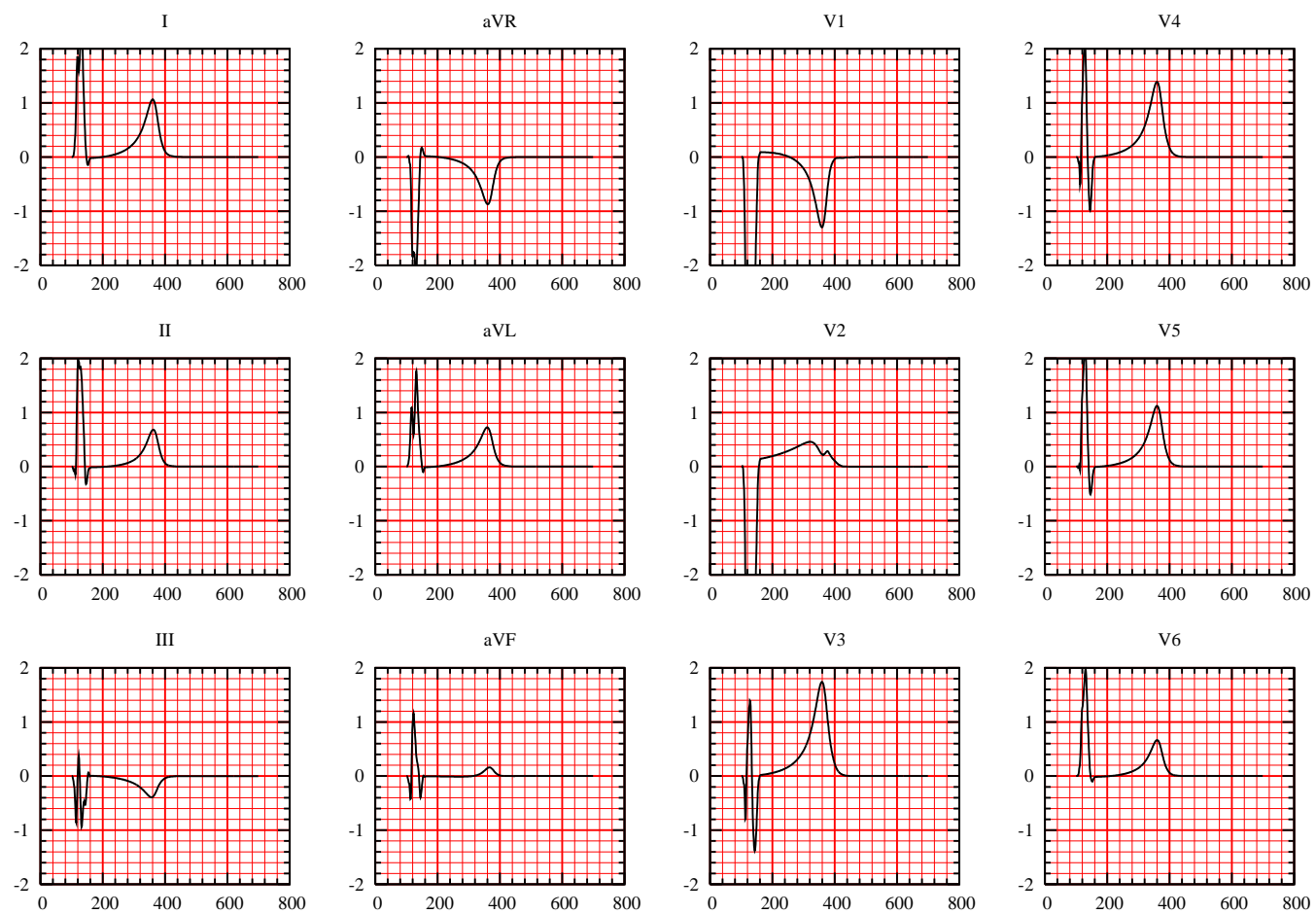

Fig. 1. An example of a computational ECG obtained with the model of $\S 2$; $x$-axis: time (milliseconds), $y$-axis: potential (millivolts).

\section{Metamodel}

As explained in the introduction, our purpose is to build a "metamodel", denoted by $f$ which takes in input an EGM and provides in output the first lead (for example) of a standard ECG. More precisely, the EGM is made of the measurements $\left(x_{i}\right)_{i=1 . . n} \in\left(\mathbb{R}^{m_{P}}\right)^{n}$ of the potential $u_{\mathrm{e}}$ in $m_{P}$ points $\left(P_{k}\right)_{k=1 . . m_{P}}$ of the heart at times $t_{1}, \ldots, t_{n}$. The first lead of the ECG $\left(y_{i}\right)_{i=1 . . n}$ is made of the difference of the potential $u_{\mathrm{T}}$ between two points of the torso denoted by $P_{L}$ and $P_{R}$. For simplicity, we first assume that $t_{1}, \ldots, t_{n}$ correspond to one heart beat (see Remark 1 below). For $k \in \mathbb{N}, 0 \leq k<n$, we define by periodicity $x_{n+k}=x_{k}$. and $x_{-k}=x_{n-k}$. 
The model presented in the previous section will be used to train and test the metamodel $f$. With the above notations, we have:

$$
x_{i}=\left(u_{\mathrm{e}}\left(t_{i}, P_{j}\right)\right)_{j=1 . . m_{P}} \in \mathbb{R}^{m_{P}},
$$

and

$$
y_{i}=u_{\mathrm{T}}\left(t_{i}, P_{L}\right)-u_{\mathrm{T}}\left(t_{i}, P_{R}\right) \in \mathbb{R}
$$

\subsection{Temporal dynamics}

Searching a function $f$ such that $y_{i} \approx f\left(x_{i}\right)$ would assume the relationship between the torso potential and the extracellular potential in the myocardium be static, which is obviously not the case (see Remark 3 below). To take into account in a simple way the time dynamics of the problem, we define a temporal window of length $m_{W}$ and we build the metamodel $f$ such that $y_{i} \approx f\left(\mathbf{x}_{i}\right)$, with

$$
\mathbf{x}_{i}=\left(x_{i}, \ldots, x_{i+m_{W}-1}\right) \in \mathbb{R}^{m_{P} \times m_{W}} .
$$

In the sequel, we denote by $m$ the dimension of the variable in the data space. We have:

$$
m=m_{P} \times m_{W} .
$$

Remark 1. When several heart beats are used to train the algorithm, we first build the vectors $\mathbf{x}_{i}$ according to (6) for each heart beat and we put together all the $\mathbf{x}_{i}$ in the training set. $\diamond$

\subsection{Kernel ridge regression}

The proposed method is known as kernel ridge regression (KRR, see [8]). It is an alternative to Support Vector Regression, an adaptation of the Support Vector Machine principle to regression problems (see e.g. [9]). In KRR, the regression function depends on all the training examples and not only on a subset (the support vectors). This typically makes the kernel ridge regression function more expensive to use but less difficult to construct than support vector regression models.

Function $f$ is looked for in a Hilbert space $\left(\mathcal{H},\langle\cdot, \cdot\rangle_{\mathcal{H}}\right)$, using a least square method with a regularization term ensuring that the norm $\|f\|_{\mathcal{H}}=\langle f, f\rangle_{\mathcal{H}}^{\frac{1}{2}}$ remains "small". We choose the space $\mathcal{H}$ as the Reproducing Kernel Hilbert Space (RKHS) based on a Gaussian kernel

$$
K(\mathbf{x}, \mathbf{y})=e^{-\frac{|\mathbf{x}-\mathbf{y}|^{2}}{2 \sigma^{2}}}, \quad \forall \mathbf{x}, \mathbf{y} \in \mathbb{R}^{m} .
$$

The kernel is said to be reproducing on $\mathcal{H}$ because of the following property:

$$
f(\mathbf{x})=\langle f(\cdot), K(\cdot, \mathbf{x})\rangle_{\mathcal{H}}, \quad f \in \mathcal{H}, \quad \forall \mathbf{x} \in \mathbb{R}^{m},
$$

where $\langle\cdot, \cdot\rangle_{\mathcal{H}}$ is the inner product in $\mathcal{H}$. The use of the Gaussian kernel can be motivated by the following property (see [10]): given a compact subset $K$ of $\mathbb{R}^{m}$, the set of the restriction to $K$ of functions from $\mathcal{H}$ is dense in the set of continuous functions from $K$ to $\mathbb{R}$. In other word, $\mathcal{H}$ is rich enough to approximate any possible continuous mapping from the EGM to a given lead of the ECG.

Consider in $\mathcal{H}$ the following regularized least square problem: given a training set $\left\{\left(\mathbf{x}_{i}, y_{i}\right)\right\}_{i=1}^{n} \in$ $\left(\mathbb{R}^{m} \times \mathbb{R}\right)^{n}$, solve

$$
\min _{f \in \mathcal{H}}\left\{\frac{1}{n} \sum_{i=1}^{n}\left(f\left(\mathbf{x}_{i}\right)-y_{i}\right)^{2}+\lambda\|f\|_{\mathcal{H}}^{2}\right\},
$$


where $\lambda>0$ is a given regularization coefficient. The representer theorem ([9]) states that the solution to $(8)$ can be written as:

$$
\sum_{i=1}^{n} \alpha_{i} K\left(\cdot, \mathbf{x}_{i}\right)
$$

where $\alpha=\left(\alpha_{i}\right)_{i=1 \ldots n} \in \mathbb{R}^{n}$ is the new unknown. Defining the Gram matrix $\mathrm{K}=\left(K_{i j}\right)_{i, j=1 \ldots n}$ with $K_{i j}=K\left(\mathbf{x}_{i}, \mathbf{x}_{j}\right)$, the training problem (8) is equivalent to solving:

$$
\min _{\alpha \in \mathbb{R}^{n}}\left\{\frac{1}{n}(\mathrm{~K} \alpha-y)^{T}(\mathrm{~K} \alpha-y)+\lambda \alpha^{T} \mathrm{~K} \alpha\right\},
$$

whose solution is simply given by

$$
\alpha=(\mathrm{K}+\lambda n \mathrm{I})^{-1} y
$$

Remark 2. If the eight independent leads of the standard ECG are to be reconstructed, one should solve eight least square problems of this kind, with different right-hand sides but with the same matrix. In other words, the training phase of the algorithm consists in solving:

$$
\mathrm{A}=(\mathrm{K}+\lambda n \mathrm{I})^{-1} \mathrm{Y}
$$

with $\mathrm{A} \in \mathbb{R}^{n \times 8}$ and $\mathrm{Y} \in \mathbb{R}^{n \times 8}$. Each column of $\mathrm{A}$ and $\mathrm{Y}$ corresponding, respectively to the coefficient $\alpha$ and to the values of a given lead of the ECG. $\diamond$

Once the training phase is completed, the reconstruction of an ECG from a given one beat EGM $\left(\tilde{x}_{i}\right)_{i=1 . . \tilde{n}} \in\left(\mathbb{R}^{m_{P}}\right)^{\tilde{n}}$ is obtained, according to $(7)$, by

$$
\widetilde{\mathrm{Y}}=\widetilde{\mathrm{K}} \mathrm{A},
$$

with $\widetilde{\mathrm{K}}_{i j}=K\left(\tilde{\mathbf{x}}_{i}, \mathbf{x}_{j}\right), i=1 \ldots \tilde{n}, j=1 \ldots n$, where the $\tilde{\mathbf{x}}_{i}$ are defined from the $\tilde{x}_{i}$ as explained in (6).

\subsection{Parameters optimization}

The proposed algorithm depends on a relatively small number of parameters: the regularization coefficient $\lambda$, the width of the Gaussian kernel $\sigma$ and the size of the temporal window $m_{W}$. Assuming that $m_{W}$ is fixed, we determine parameters $\lambda$ and $\sigma$ with a leave-one-out procedure: we train the metamodel with all the examples but one and we assess its performance on the example which has been removed from the training set. In other words, we use of cross validation with a singleton as testing set. We repeat the procedure with all the singletons of the training set. The brute force leaveone-out may be extremely expensive. Fortunately, there exists formulae to compute the leave-one-out mean-squared error using the results of a single training on the whole training set. This is one of the advantages of the kernel ridge regression over support vector regression. We refer for example to [6] for more details.

\section{Results}

\subsection{Synthetic Data}

We use the mathematical model presented in $\S 2$ to generate "synthetic" data. We consider the extracellular potential in $m_{P}=5$ points given in the heart (see Fig. 2) and the DI lead of the corresponding computational ECG. The versatility of the numerical model allows to test the reconstruction algorithm in various situations more easily than with clinical data. All the examples of this section are obtained with the following parameters: $\sigma=150, \lambda=10^{-4}, m_{W}=6$ ( $\sigma$ and $\lambda$ being automatically given by the leave-one-out procedure briefly described in $\S 3.3)$. 

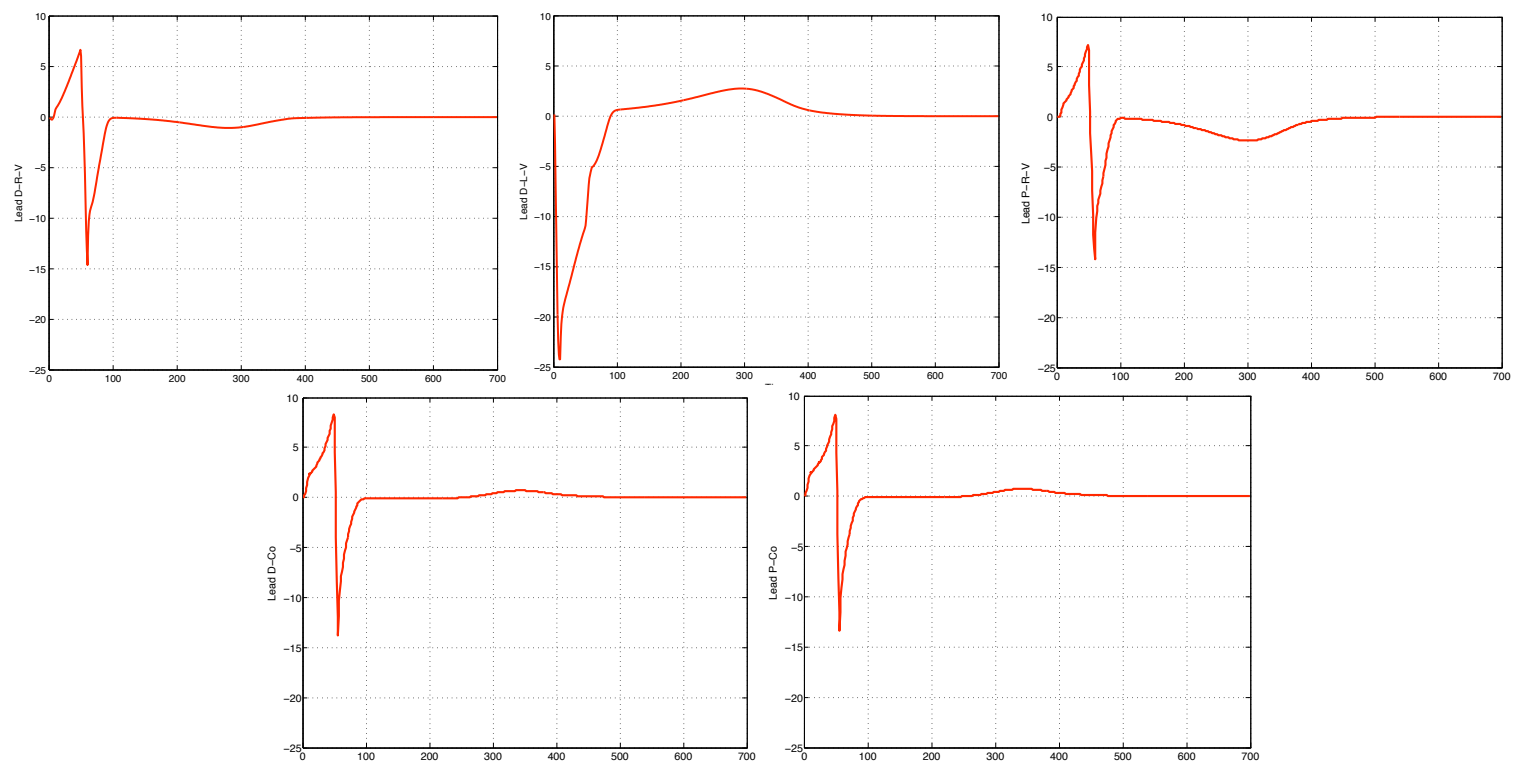

Fig. 2. An example of a computational EGM obtained with the model of $\S 2$. Here, the $m_{p}=5$ points are located (from left to right and top to bottom) in distal RV, distal LV, proximal RV, distal coil, proximal coil.

We focus on a pathological case called right bundle branch block (RBBB). Starting from the healthy situation presented in Fig. 1, we simulate a RBBB by artificially delaying the activation of the right ventricle. For example, a delay of $10 \mathrm{~ms}$ will be denoted by RBBB-10. A larger delay of course corresponds to a situation which is farther from the healthy state.

First suppose the test case is a part of the training set. In that case, the reconstruction of the ECG is, as expected, very good. In Fig. 3, we see that increasing the size of the training set does not deteriorate significantly the result.
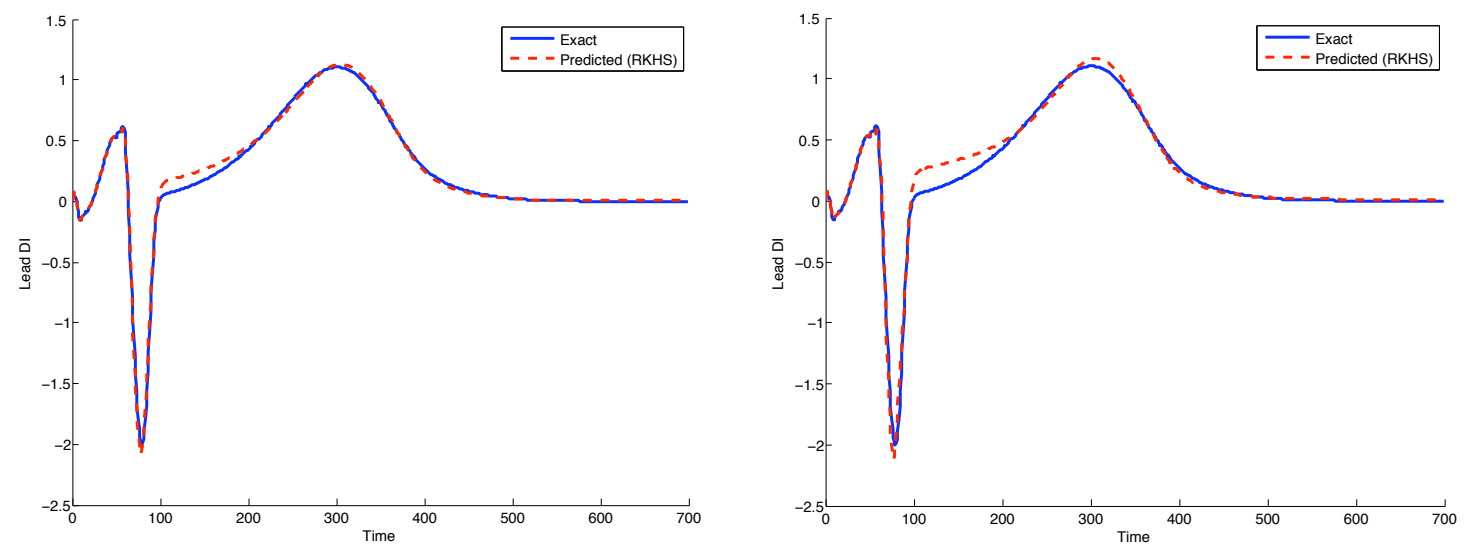

Fig. 3. Reconstruction of a RBBB-50 when the training set is RBBB- $\{10,50,100,200\}$ (left) and RBBB- $\{10$, 50, 100, 200, 400, 700\} (right). 
Remark 3. We show in Fig. 4 that the results obtained with $m_{W}=6$ are clearly better than those obtained with $m_{W}=1$. This confirms that the relationship EGM/ECG is not static and that the technique of temporal windows is a possible solution to handle the dynamic of the problem. The optimal length of the temporal window could be determined, as for $\sigma$ and $\lambda$, using the leave-one-out procedure. $\diamond$
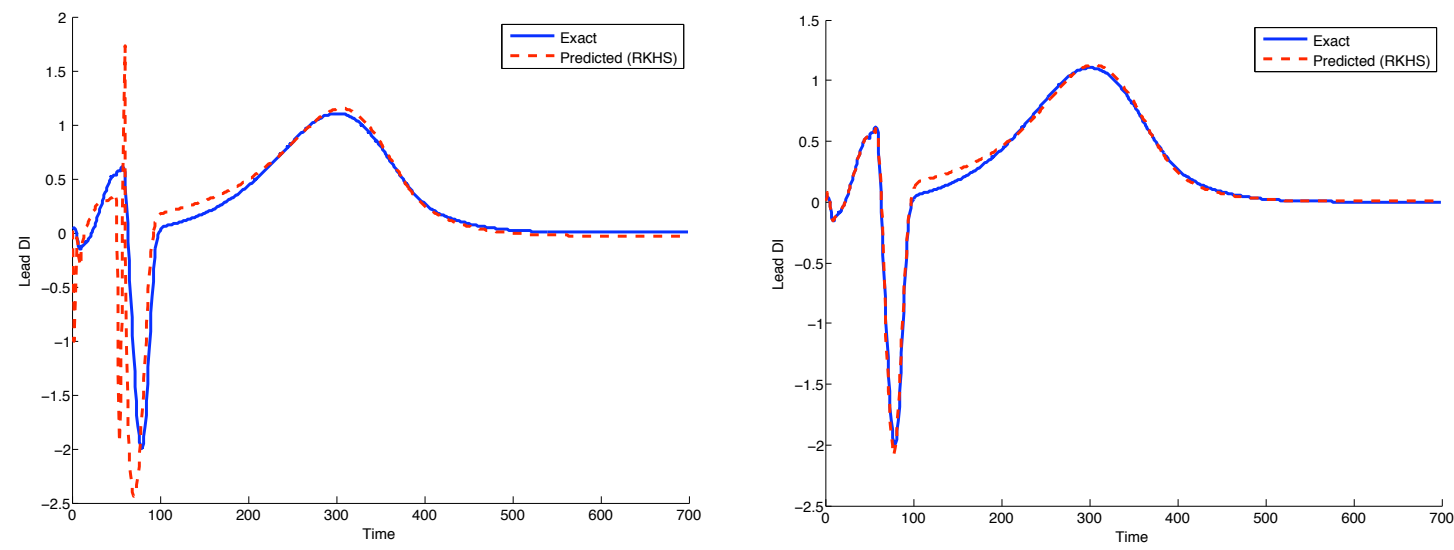

Fig. 4. Comparison of reconstruction of a RBBB-50 when the training set is RBBB- $\{10,50,100,200\}$ using different time window parameter: Left, $m_{W}=1$. Right, $m_{W}=6$.

Next consider a test case which does not belong to the training set. In practice, this corresponds for example to the following situation: the patient has a slight right bundle branch block when the algorithm is trained, but when he comes back a few months later, the pathology is getting worse. Fig. 5 (left) shows what happens if we train the algorithm with a RBBB-10, and if we try to reconstruct the ECG corresponding to a RBBB-50. We can see that the result is poor. Since we get good results
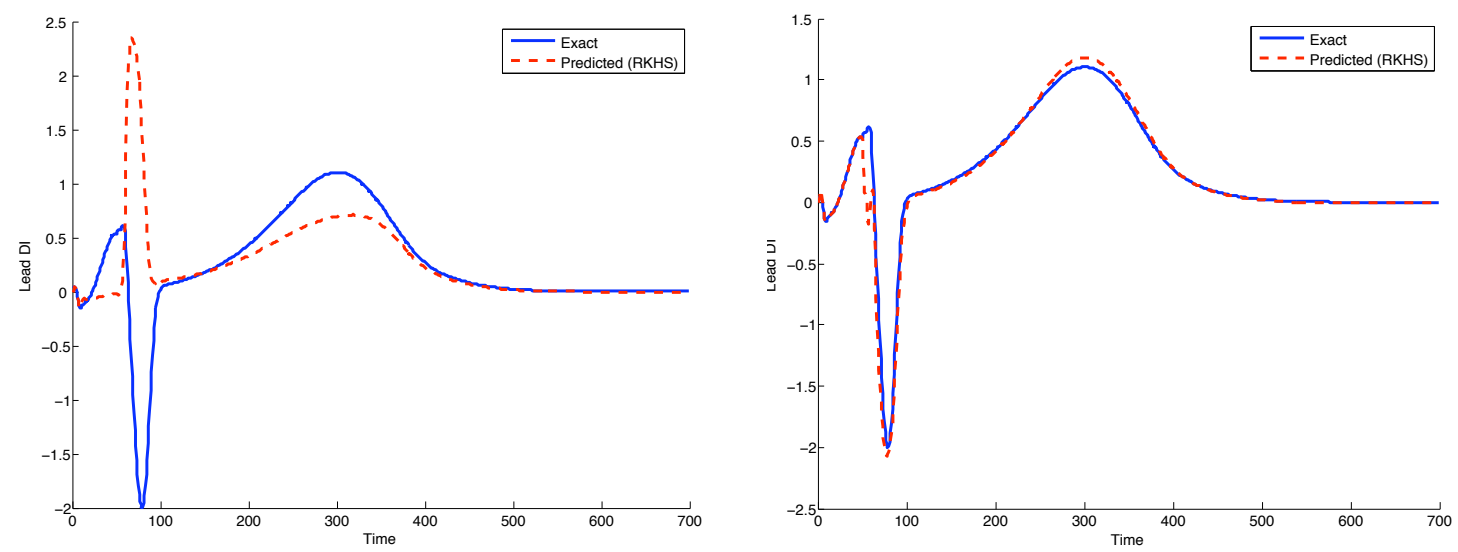

Fig. 5. Left: Reconstruction of a RBBB-50 with training from RBBB-10. Right: Reconstruction of a RBBB-50 when the training set is RBBB- $\{10,40,60\}$ (right)

with larger training sets, a natural idea is to enrich the training set so that the new situation be close 
to a training case. For the sake of illustration, suppose a patient has a RBBB-10 and suppose the numerical model can be parametrized to reproduce the patient's ECG. With the simulation tool, we can investigate possible situations the patient may encounter in the future. For example, assume we compute a RBBB-40 and a RBBB-60. Thus we build a training set made of RBBB- $\{10,40,60\}$. We show in Fig. 5 (right) that the prediction obtained if the patient has a RBBB-50 is then much better.
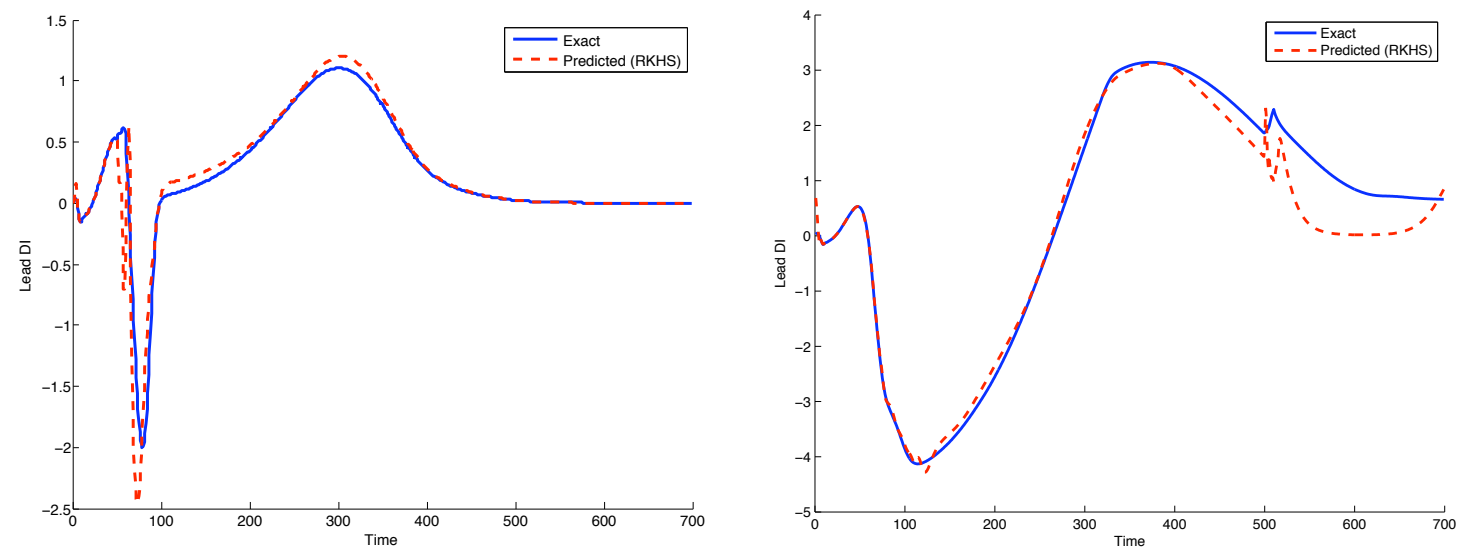

Fig. 6. Reconstruction of a RBBB-50 (left) and a RBBB-500 (right) when the training set is RBBB- $\{10,20$, 40, 60, 70, 80, 100, 150, 200,300,400,700\}.

In Fig. 6 (Left), we show the result obtained with a much larger training set RBBB-10, 20, 40, $60,70,80,90,100,120,150,200,300,400,700\}$. In that case the result for a RBBB-50 is slightly less accurate than with the training set of only 3 cases, but the resulting metamodel is more robust and can be used in a wide range of situations. This is illustrated in Fig. 6 (right), where we report the reconstruction of a RBBB-500 with the same training set.

\subsection{Clinical Data}

In this paragraph we use our method to reconstruct ECGs from clinical EGMs. In Fig. 7 (left) we reconstruct the ECG of a myocardial ischemia disease patient ("patient 1"), with a training done on a Tachycardia heart disease patient ("patient 2"). As expected the reconstruction is totally wrong which confirms that the metamodel has to be patient specific. In Fig. 7 (right), the data of "patient 1 " is added to the training set and the reconstruction becomes very good. Again, if we add more cases, for example an atrial fibrillation disease ("patient 3") and a LBBB disease ("patient 4") in the traning data set, the reconstruction of the ECG remains acceptable (Fig. 8, left). This confirms on clinical data the trend observed with numerical simulations. We have also observed that the results are usually very good when the reconstruction is performed on a heart beat different from those used for the training on the same patient (Fig. 8, right)

\section{Conclusion}

In this work we have considered a machine learning approach, based on the kernel ridge regression method, in order to provide ECG information from a set of EGM measurements.

The procedure has been trained using synthetic data from numerical simulations, based on a 3D mathematical model of the ECG involving a quite complete description of the electrical activity of the heart and the torso. A few clinical data have also been considered for illustration. Several examples 

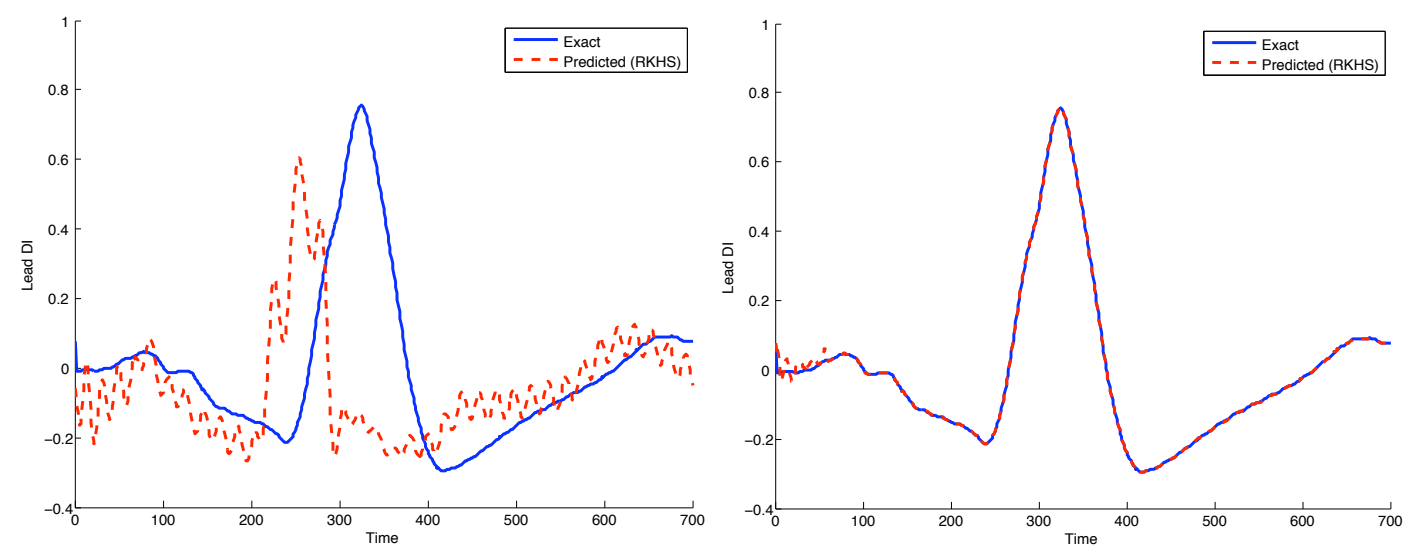

Fig. 7. Reconstruction of "patient 1" ECG when the training set is recorded from: "patient 2" (left) and "patient 1" \& "patient 2" (right).
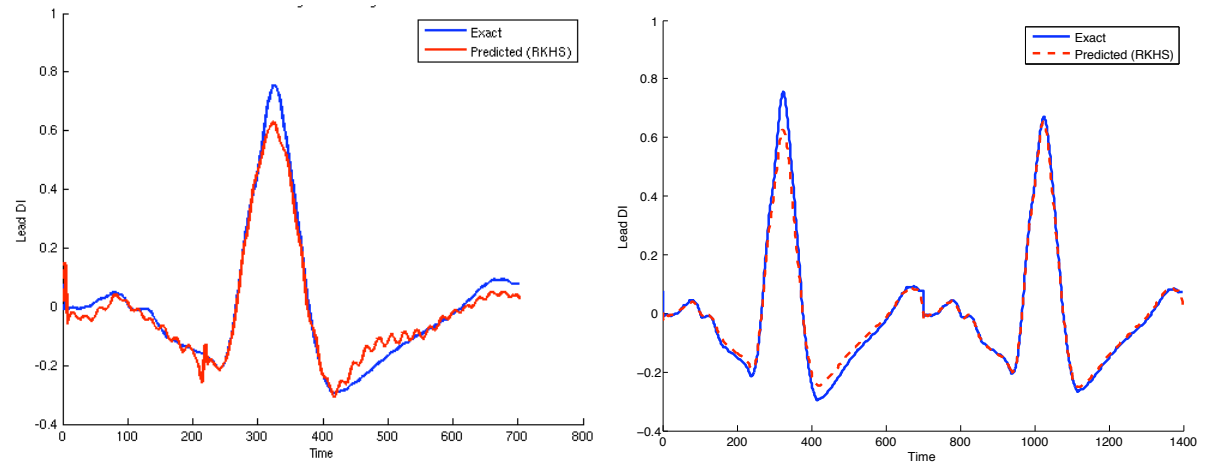

Fig. 8. Left: Reconstruction of "patient 1" ECG when the training set is recorded from "patient 1", "patient 2", "patient 3" and "patient 4". Right: Reconstruction of a two-heart-beat "patient 1" ECG when the training is performed on different heart beats of the same patient (right). 
showed the method is able to reconstruct conveniently the ECG information included in the training data set, and is robust when reconstructing situations close to the training data set. In the proposed approach, the solution depends on all training examples, which may be too expensive. This point could be improved in future works.

We have also illustrated that a possible use of the numerical simulation could be to enrich available clinical data to conveniently investigate a wider range of situations. This strategy nevertheless relies on the capability to adapt the model to a specific patient, which is the topic of ongoing researches.

Acknowledgment: This work was partially supported by ELA Medical ${ }^{4}$, a Sorin group company, and by the CardioSense $3 D^{5}$ project (INRIA). We are thankful to Alain Ripart and Renzo Dal Molin for introducing us to this problem and for many interesting discussions.

\section{References}

1. M. Boulakia, M.A. Fernández, J.-F. Gerbeau, and N. Zemzemi. Towards the numerical simulation of electrocardiograms. In F.B. Sachse and G. Seemann, editors, Functional Imaging and Modeling of the Heart, number 4466 in Lecture Notes in Computer Science, pages 240-249. Springer-Verlag, 2007.

2. M. Boulakia, M.A. Fernández, J.-F. Gerbeau, and N. Zemzemi. A coupled system of PDEs and ODEs arising in electrocardiograms modelling. Applied Math. Res. Exp., 2008. doi:10.1093/amrx/abn002.

3. J. Malmivuo and R. Plonsey. Bioelectromagnetism. principles and applications of bioelectric and biomagnetic fields. Oxford University Press, New York, 1995.

4. C.C. Mitchell and D.G. Schaeffer. A two-current model for the dynamics of cardiac membrane. Bulletin Math. Bio., (65):767-793, 2003.

5. J.S. Nagumo, S. Arimoto, and S. Yoshizawa. An active pulse transmission line stimulating nerve axon. Proc. IRE, (50):2061-2071, 1962.

6. M. Opper and O. Winther. Advances in Large-Margin Classifiers, chapter 17. Gaussian Processes and SVM: Mean Field and Leave-One-Out. The MIT Press, 2000.

7. F.B. Sachse. Computational Cardiology: Modeling of Anatomy, Electrophysiology, and Mechanics. Springer-Verlag, 2004.

8. Craig Saunders, Alexander Gammerman, and Volodya Vovk. Ridge regression learning algorithm in dual variables. In Proceedings of the Fifteenth International Conference on Machine Learning (ICML'98), pages 515-521, Madison, Wisconsin, USA, July 1998.

9. B. Scholkopf and A.J. Smola. Learning with Kernels: Support Vector Machines, Regularization, Optimization, and Beyond. MIT Press, Cambridge, MA, USA, 2001.

10. Ingo Steinwart. On the influence of the kernel on the consistency of support vector machines. Journal of Machine Learning Research, 2:67-93, November 2001.

11. J. Sundnes, G.T. Lines, X. Cai, B.F. Nielsen, K.-A. Mardal, and A. Tveito. Computing the electrical activity in the heart. Springer-Verlag, 2006.

\footnotetext{
${ }^{4}$ http://www.elamedical.com

${ }^{5}$ http://www.inria.fr/CardioSense3D
} 